\title{
Dialektisch-behaviorale Therapie nach der Akutphase - die Herausforderung annehmen, das System zu verlassen (DBT-ACES)
}

\author{
Klaus Hoeschel $^{\mathrm{a}}$ Stephanie Pfluegler ${ }^{\mathrm{a}} \quad$ Mariel Rinke $^{\mathrm{a}} \quad$ Kerstin Burmeister $^{\mathrm{a}}$ \\ Christos Chrysanthou ${ }^{\mathrm{a}}$ Katherine A. Comtois ${ }^{\mathrm{b}}$ \\ ${ }^{a}$ Abteilung für Allgemeine Psychiatrie und Psychotherapie, LWL-Klinik Lengerich, Deutschland \\ ${ }^{\mathrm{b}}$ Harborview Medical Center, University of Washington, Seattle, WA, USA
}

\section{Schlüsselwörter}

Borderline-Persönlichkeitsstörung .

Kognitiv-behaviorale Therapie - Psychotherapie .

Dialektisch-behaviorale Therapie

\section{Zusammenfassung}

Neuere Verlaufsuntersuchungen bei Patienten mit Borderline-Persönlichkeitsstörungen zeigen, dass die akute Symptomatik, wie z.B. Suizidversuche, Selbstverletzungen oder impulsive Verhaltensweisen, starken Schwankungen unterliegt. Emotionale Probleme, wie andauernde Depressivität oder Ärgergefühle, Identitätsstörungen, Defizite beim Erreichen sozialer Ziele und eine ungenügende Lebenszufriedenheit sowie körperliche Langzeitschäden mit Schmerzsyndromen oder metabolischen Syndromen, haben ein hohes Chronifizierungsrisiko. Die verhaltenstherapeutische Behandlung mit dialektisch-behavioraler Therapie (DBT) verfügt heute zwar über ausreichende empirische Wirksamkeitsnachweise, das Behandlungsmanual bezieht sich aber vorwiegend auf Interventionen in der ersten Behandlungsstufe, während die Arbeit in nachfolgenden Behandlungsstufen noch nicht ausreichend manualisiert und überprüft ist. Mit einem Behandlungsmodell, das 2 einjährige Behandlungsphasen der DBT vorsieht, wird derzeit ein von der Arbeitsgruppe um K.A. Comtois in Seattle entwickeltes Modell (DBT-ACES) erprobt. Darin ist nach dem Abklingen der akuten Symptomatik im ersten Behandlungsjahr ein zweites Behandlungsjahr vorgesehen, in dem der Fokus auf dem Erreichen einer sozialen Integration mit der Aufnahme von Arbeitstätigkeit, dem Aufbau normativer sozialer Netze sowie der Förderung von Unabhängigkeit von Leistungen des Gesundheits- und Sozialsystems liegt. Erste Daten aus einer amerikanischen Untersuchung belegen die Wirksamkeit des Ansatzes DBT-ACES. Dieser Artikel skizziert das Vorgehen von DBT-ACES, das derzeit auch in Deutschland geprüft wird.

\author{
Keywords \\ Borderline personality disorder . \\ Cognitive behavioral therapy - Psychotherapy . \\ Dialectic behavioral therapy
}

\section{Summary}

Dialectic Behavioral Therapy - The Concept of Accepting the Challenges of Exiting the System (DBT-ACES)

Recent process analyses on patients with borderline personality disorder (BPD) show that the acute symptomatology apparently is subject to considerable fluctuations, for example suicidal, self-harming or impulsive behavior. Emotional problems, like a continuing depressive mood or feelings of anger, identity disturbances, lack of skills in reaching social goals and insufficient life satisfaction as well as longterm injuries such as pain syndromes or metabolic syndromes all have a high risk to become chronic.

Today, the psychotherapeutic treatment with dialectic behavioral therapy (DBT) provides ample empiric evidence of efficacy in clinical work with BPD patients; but the manual focuses predominantly on interventions at the first stage of treatment. All following stages have not been sufficiently researched and manualized yet. Developed by the team of K.A. Comtois in Seattle, a 2-year DBT program (DBT-ACES) is currently being tested. The first year of the program deals with acute symptoms, the second year concentrates on reaching the goal of social integration with finding paid work, establishing normative social networks and becoming independent of the health and social system. Already some data from an American research project show the efficacy of DBT-ACES. The approach of DBT-ACES, which is also tested in Germany, is outlined in the following article.

\section{KARGER \\ Fax +497614520714 \\ Information@Karger.de}

www.karger.com (c) 2011 S. Karger GmbH, Freiburg

$1016-6262 / 11 / 0214-0239 \$ 38.00 / 0$

Accessible online at:

www.karger.com/ve
Dr. Klaus Hoeschel

LWL-Klinik Lengerich

Abteilung für Psychiatrie und Psychotherapie

Parkallee 10, 49525 Lengerich, Deutschland

Tel. +49 548 112-336, Fax -484

hoeschel@t-online.de 
Das Störungsbild der

Borderline-Persönlichkeitsstörung

In repräsentativen Untersuchungen findet man im Bevölkerungsquerschnitt der europäischen und amerikanischen Bevölkerung unter Erwachsenen einen Anteil von 0,7 bis 1,4\%, bei denen die Kriterien der Borderline-Persönlichkeitsstörung (BPS) erfüllt sind [Coid et al., 2006; Lenzenweger et al., 2007], die Lebenszeitprävalenz beläuft sich auf 3\% [Trull et al., 2010]. Wenn 5 von 9 Kriterien auf einen Patienten eindeutig zutreffen wird die Diagnose nach dem Klassifikationssystem des Diagnostic and Statistical Manual of Mental Disorders (DSM-IV) gestellt. Dabei können affektive Kriterien (Probleme mit Wutgefühlen, chronische innere Leere, affektive Instabilität), kognitive Kriterien (paranoide Vorstellungen und dissoziative Symptome, Identitätsstörungen), Verhaltenskriterien (suizidale und parasuizidale Handlungen oder Drohungen, impulsives Verhalten) und zwischenmenschliche Kriterien (Angst vor dem Alleinsein und Verlassenwerden, instabile Beziehungen) unterschieden werden.

Die Mehrzahl der Patienten mit BPS suchen aktiv psychiatrische oder psychotherapeutische Behandlungen auf, die jedoch häufig nicht von durchschlagendem Erfolg gekrönt sind. Gemäß einer neuen Metaanalyse von Barnicot und Mitarbeitern [Barnicot et al., 2011] liegen die Behandlungsabbruchraten bei Borderline-Patienten in Therapien mit wissenschaftlichem Wirksamkeitsnachweis bei durchschnittlich 25-30\%. Es ist zu befürchten, dass weniger spezialisierte Therapien zu noch höheren Therapieabbruchraten führen; so berichtete die Freiburger Arbeitsgruppe vor fast 15 Jahren, dass 75\% der behandlungsuchenden Borderline-Patienten die Behandlung abbrachen [Jerschke et al., 1998]. Die resultierenden Behandlungskosten pro Patient und Jahr wurden 1998 auf 17000 EUR geschätzt, von denen etwa 16000 EUR auf den stationären Bereich entfielen. Auch in neueren Untersuchungen [Soeteman et al., 2008; Van Asselt et al., 2007] werden die Behandlungskosten von behandlungssuchenden Patienten mit BPS auf Beträge zwischen 11000 EUR und 17000 EUR pro Patient und Jahr geschätzt.

Trotz dieser hohen Inanspruchnahme von Leistungen des Gesundheitssystems weist der Verlauf der Störung bei der Mehrzahl der Betroffenen starke Schwankungen auf. Die Arbeitsgruppe um Zanarini [Zanarini et al. 2010a] beobachtete in einer Langzeitverlaufsstudie, dass nach 2 Jahren etwa 35\% der diagnostizierten Borderline-Patienten nicht mehr die diagnostischen Kriterien der BPS erfüllten; nach weiteren 2 Jahren erfüllten aber etwa die Hälfte der zunächst eine Besserung zeigenden Patienten wieder die vollständigen Kriterien des Störungsbilds. Es kommt hinzu, dass bestimmte Verhaltensweisen, wie suizidales und parasuizidales Verhalten, sich eher zu bessern scheinen als depressive Störungen, Wutgefühle und tiefgreifende Gefühle von Einsamkeit und Verlassenheit [Zanarini et al., 2007]. Noch ungünstiger scheint der Verlauf des sozialen Funktionsniveaus zu sein. Zanarini und Mitarbei- ter [Zanarini et al., 2010b] berichten, dass bei einer stationären Aufnahme zu einer kurzen Krisenintervention nur 1/4 der Boderline-Patienten über ein gutes soziales Funktionsniveau verfügen. Innerhalb von 2 Jahren nach der Krisenintervention hatten $70 \%$ der Patienten mit initial gutem Funktionsniveau dieses verloren, während nur $15 \%$ der Patienten mit initial schlechtem sozialem Funktionsniveau das Kriterium des guten Funktionsniveaus erreichten. Winograd und Mitarbeiter [Winograd et al., 2008] untersuchten Jugendliche im Alter von 14 Jahren auf das Vorliegen von Merkmalen einer BPS. 20 Jahre später wurde das Vorliegen von BPS-Merkmalen und das soziale Anpassungsniveau erneut erhoben. Es zeigte sich, dass die typischen BPS-Merkmale in diesem Zeitraum bei den meisten im Jugendalter Betroffenen deutlich zurückgegangen waren. Weiterhin defizitär blieb jedoch das soziale Anpassungsniveau (sowohl die Lebenszufriedenheit, die Qualität von Partnerbeziehungen und das Ausmaß sozialer Unterstützung als auch das Erreichen von Ausbildungs- und Berufszielen), auch wenn die Borderline-Symptomatik sich bis zum Erwachsenenalter verbessert hatte. Auch wenn die Übertragung der Ergebnisse dieser beiden amerikanischen Untersuchungen auf europäische Verhältnisse nicht unproblematisch ist, lässt sich ein hohes Chronifizierungsrisiko der psychopathologischen Symptomatik befürchten. Das Risiko einer schlechten sozialen Anpassung mit Arbeitslosigkeit und ungenügender Integration in soziale Netze scheint unabhängig vom Verlauf der psychopathologischen Symptomatik deutlich erhöht zu sein, wenn im Jugendalter Boderline-Merkmale bestehen. Darüber hinaus scheint das Risiko von chronischen und schwer behandelbaren körperlichen Erkrankungen (Fibromyalgie, Erschöpfungssyndrome, Adipositas, Diabetes, Bluthochdruck oder Schmerzsyndrome) im höheren Alter bei Patienten mit BPS deutlich erhöht zu sein [Zanarini et al., 2005]. Schließlich suizidieren sich 4-10\% der Betroffenen [Paris und ZweigFrank, 2001; Zanarini et al., 2010a].

\section{Die Behandlung der BPS mit DBT}

Bei Patienten mit BPS gilt es also, diese nach erfolgter Diagnostik möglichst frühzeitig einer störungsspezifischen Therapie zuzuführen, die neben der direkten Behandlung der psychopathologischen Symptomatik auch das Gesundheitsverhalten und die soziale Integration der Patienten einschließt.

Mit speziell auf die Bedürfnisse von Patienten mit BPS abgestimmten Behandlungsmethoden unterscheidet Linehan [1993a] mehrere Behandlungsstufen («Stages») der DBT (Tab. 1). In der ersten Behandlungsstufe («Stage I») zielen die Interventionen auf eine Reduktion krisengenerierenden und therapiegefährdenden Verhaltens (vorrangig suizidales, parasuizidales, selbstverletzendes und massiv therapiegefährdendes Verhalten) sowie auf den Aufbau grundlegender Verhaltensfertigkeiten («Skills»). Die Behandlungsmethodik ist manualisiert [Linehan, 1993a,b; Bohus und Wolf, 2009] 
Tab. 1. Die von Linehan $[1993$ a,b] in der dialektisch behavioralen Therapie vorgesehen Behandlungsstadien (Stages) zur Behandlung von Patienten mit BPS

\begin{tabular}{cl}
\hline Stage & Ziele \\
\hline I & - Verringerung von krisengenerierendem Verhalten \\
& - Verringerung von therapiegefährdendem Verhalten \\
& - Verringerung von krisengenerierenden Umweltbedingungen \\
& - Aufbau grundlegender Fertigkeiten \\
& - Verbesserung posttraumatischer Erlebens- und Reaktionsmuster \\
II & - Aufbau eines nicht-traumatisierenden Erlebens von Gefühlen \\
& - Aufbau eines nicht-traumatisiereden Kontaktes mit der Umwelt \\
& - Verbesserung von Problemen in der Lebensführung und Lebensqualität \\
III & - Verbesserung des Selbstvertrauens \\
& - Verbesserung des Zugehörigkeitsgefühls \\
& - Verbesserung der Gefühle von Freiheit und Freude \\
IV & - Annähern an eine spirituelle Erfüllung \\
&
\end{tabular}

und wird ambulant mit einem Einzelkontakt und einem zweistündigen Gruppenskillstraining pro Woche sowie der Möglichkeit für Telefonanrufe angeboten. Zusätzlich versteht die DBT sich als Behandlungsmethode, die im Team stattfindet, so dass wöchentliche Teamsitzungen für DBT-Therapeuten obligatorisch sind.

Im Gegensatz zu den sehr konkreten, ausgezeichnet operationalisierten und evaluierten Behandlungsstrategien in Stage I wurden Manuale für die «Stage II»-Therapie erst in den vergangenen Jahren entwickelt und evaluiert [z.B. Steil et al., 2011]. In Stage II ist es das Ziel, traumatisierende Erlebensund Reaktionsmuster zu verbessern und den Patienten ein nicht-traumatisches Erleben von Gefühlen und einen nichttraumatisierenden Kontakt mit der Umwelt zu ermöglichen. In der dritten Behandlungsstufe («Stage III») werden die Patienten dann unterstützt, mit Hilfe der erlernten Fertigkeiten Probleme in der Lebensführung zu verbessern und das Selbstbewusstsein und das Zugehörigkeitsgefühl zu stärken. Die vierte Behandlungsstufe («Stage IV») bezieht sich dann auf den Aufbau von Gefühlen von Freiheit, Freude und spiritueller Erfüllung.

K.A. Comtois arbeitete mit ihrer Arbeitsgruppe am Harborview Medical Center in Seattle ein noch unpubliziertes Manual für die weiterführende Behandlung der BPS in Stage III aus. Darin ist nach erfolgreicher einjähriger Behandlung der unkontrollierten psychopathologischen Symptomatik in Stage I und II ein weiterführendes Behandlungsjahr für diejenigen Patienten vorgesehen, die den Wunsch haben, die erforderlichen Fähigkeiten aufzubauen um (a) den Anforderungen einer belohnenden, strukturierten und zielgerichteten schulischen oder beruflichen Tätigkeit gerecht zu werden. Darüber hinaus werden die Ziele (b) der Unabhängigkeit vom Gesundheits- und Versorgungssystem und (c) einer Integration in normative soziale Netze sowie (d) das Erreichen persönlicher Ziele zur Steigerung der Lebensqualität gefördert. Aufgrund der Zielsetzungen des Abbaus von Abhängigkeiten von Leistungen des psychiatrischen Versorgungssystems und des Aufbaus einer subjektiven, sozialen und wirtschaftlichen Unabhängigkeit und Selbständigkeit nannten sie den Ansatz
«DBT-ACES». «ACES» ist dabei ein Akronym für den Begriff «Accepting the Challenges of Exiting the System»wörtlich übersetzt geht es dabei also darum, die Herausforderung anzunehmen, das (psychiatrische Versorgungs-)System zu verlassen.

\section{Die Behandlung nach DBT-ACES}

In dem Konzept werden zwei Behandlungsphasen unterschieden (Abb. 1). In der ersten Phase der Behandlung werden den Patienten neben einem Einzelkontakt pro Woche ein zweistündiges Gruppenskillstraining sowie die Möglichkeit zu Krisenanrufen angeboten. Eine psychiatrische Mitbehandlung kann zusätzlich aufgenommen werden. Die erste Behandlungsphase dauert maximal ein Jahr und entspricht der bekannten und manualisierten Standard-DBT-Behandlung in Stage I und II.

Wenn keine dringend behandlungsbedürftigen Stage-Ioder Stage-II-Probleme vorliegen oder ausreichend Zeit für weitere Themen ist, werden auch im ersten Behandlungsjahr Stage-III-Ziele verfolgt.

\section{Behandlungsangebote im ersten Jahr}

Die Einzeltherapie findet einmal wöchentlich für $50 \mathrm{~min}$ statt, die Einzelkontakte orientieren sich an der Beschreibung im DBT-Manual von Linehan [1993a]. Um konzepttreu nach DBT zu arbeiten, müssen die Einzeltherapeuten die Balance zwischen Akzeptanzstrategien (z.B. Validierung; zugewandte und verständnisvolle Kommunikation) und Veränderungsstrategien (z.B. auf Problemlösungen drängen, Kontingenzmanagement einsetzen, provokative und freche Kommunikation) wahren. Dabei werden typische DBT-Merkmale wie der Aufbau dialektischer Betrachtungsweisen realisiert, wie z.B. «Sowohl-als-auch-Denken» anstelle von «Schwarz-Weiß-Denken». Eine typische DBTEinzeltherapiesitzung folgt einem strukturierten Sitzungs- 


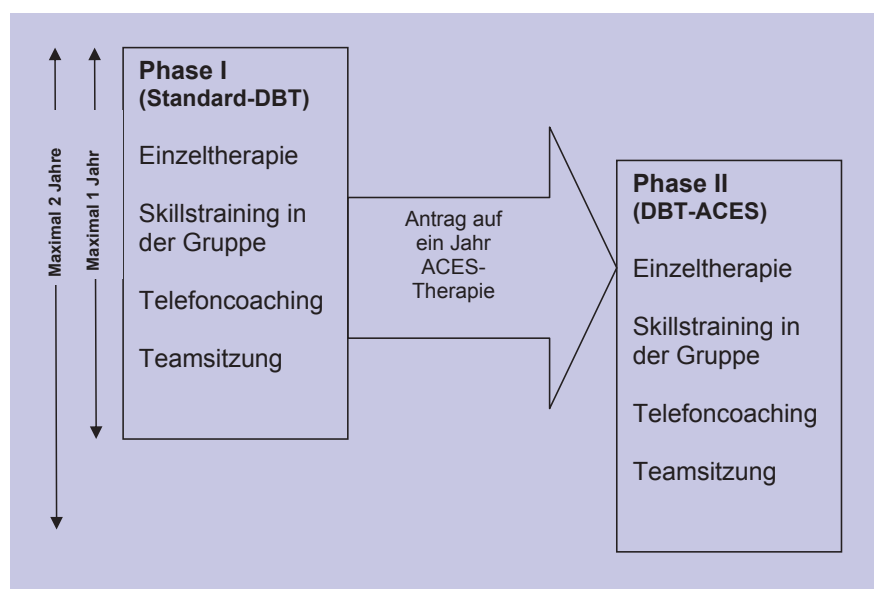

Abb. 1. Der zeitliche Ablauf des Behandlungsangebotes mit maximal einem Jahr dialektisch-behavioraler Therapie (Standard-DBT) im ersten Jahr und der Möglichkeit zum Wechsel in das zweite Behandlungsjahr nach dem ACES-Konzept.

ablauf mit einer Sichtung der Tagebuchkarten zu Beginn der Therapiestunde, in denen die Patienten den Drang und die Handlungen unkontrollierter problematischer Verhaltensweisen täglich protokollieren. Der Sitzungsschwerpunkt ergibt sich dann aus den Einträgen entsprechend der Gefährlichkeit von problematischen Verhaltensweisen.

Beim Telefoncoaching haben die Patienten die Möglichkeit, ihre Therapeuten unter fest vereinbarten Bedingungen und unter Wahrung der persönlichen Grenzen des Therapeuten anzurufen. Die Telefonstrategien werden entsprechend des DBT-Manuals gestaltet, sollen möglichst kurz gehalten werden (möglichst 15 min nicht überschreiten) und sind als Hilfestellung bei der Anwendung von Skills im Alltag gedacht.

Das Skillstraining wird entsprechend der Manuale [Linehan, 1993b; Bohus und Wolf, 2009] durchgeführt. Die Patienten werden in den grundlegenden Skills der Module Stresstoleranz, bewusster Umgang mit Gefühlen, Achtsamkeit und zwischenmenschliche Fertigkeiten in einer Gruppe von maximal 8 Patienten und 2 Skillstrainern in wöchentlichen, zweistündigen Gruppensitzungen geschult.

Neben den typischen DBT-Strategien wie z.B. Validierung, Erhöhung der Selbstverpflichtung («Commitment»), Selbstbeobachtung mit Hilfe von Tagebuchkarten oder dem Aufbau von Alternativfertigkeiten ist im ersten Behandlungsjahr ein Kontingenzmanagement vorgesehen.

- Kontingenzmanagement für versäumte Sitzungen:

Patienten, die 4 aufeinanderfolgende Einzelkontakte oder 4 aufeinanderfolgende Sitzungen Skillstraining versäumen, werden von der Behandlung ausgeschlossen.

- Kontingenzmanagement für den Aufbau geplanter Aktivitäten:

Sind folgende Bedingungen über einen Zeitraum von 4 Wochen nicht erfüllt, ruht die Behandlung, bis die Bedingungen wieder für mindestens 1 Woche erfüllt sind:
Ab Monat 4: 10 h geplante Aktivitäten werden pro Woche durchgeführt.

Ab Monat 8: 15 h geplante Aktivitäten werden pro Woche durchgeführt.

Als geplante Aktivitäten zählen alle Aktivitäten (die natürlich nicht selbstschädigend sein dürfen; also z.B. Spaziergänge im Park, geplante Treffen mit Freunden, und so weiter), egal ob sie allein oder in Gesellschaft durchgeführt werden. Allerdings müssen die Aktivitäten mindestens einen Tag im Voraus geplant und protokolliert werden und unabhängig von der aktuellen Stimmung umgesetzt werden. Durch den schrittweisen Aufbau geplanter Aktivitäten können Patienten zunehmend sicherer lernen, ihre Stimmung durch das eigene Verhalten zu beeinflussen und nicht umgekehrt das Verhalten von der Stimmung abhängig zu machen. Der Aktivitätenaufbau ist ein Zusatzmodul von DBT-ACES im ersten Jahr und in der ursprünglichen manualisierten DBT nicht als fester Bestandteil vorgesehen.

\section{Der Antrag auf Teilnahme am ACES-Jahr}

Den Übergang zur zweiten Behandlungsphase (dem ACESJahr) bildet der Antrag auf das ACES-Jahr. Der Antrag wird vom Patienten gestellt und vom Einzeltherapeuten unterstützt. Der Antrag auf das ACES-Jahr wird vom Teamleiter in Kooperation mit dem Behandlungsteam geprüft. Grundsätzlich kann der Patient mit dem ACES-Jahr beginnen, wenn der Erfolg der Behandlung im ersten Jahr aus dem Antrag hervorgeht. Hierfür sind erforderlich:

- 2 Monate Freiheit von hochrangigem Stage-I-Verhalten:

Keine suizidalen oder parasuizidalen Handlungen, keine ungeplanten stationären Notfallaufnahmen, kein schwerwiegend therapiegefährdendes Verhalten.

- Abbau von weiteren Versorgungsangeboten:

Mit Ausnahme einer geregelten und sinnvollen psychiatrischen Behandlung und natürlich von notwendigen somatischen Behandlungen, sollten alle weiteren psychotherapeutischen Behandlungen oder psychosozialen Unterstützungen (Integrationshilfen, Reha-Maßnahmen usw.) bei Beginn des ACES-Jahres abgebaut sein, da die Inanspruchnahme solcher Unterstützungen dem Ziel «exiting the system» widersprechen würde.

- Ausreichende Kenntnis der grundlegenden Skills:

Die Skillskenntnis wird mit einem schriftlichen Skillstest geprüft, der Test kann in Parallelversionen so oft wiederholt werden bis er bestanden wurde.

- Ausreichende Fähigkeit, vom Gruppenskillstraining zu profitieren:

Die Effektivität der Gruppenteilnahme wird mit einer vorgedruckten Beurteilung durch einen der Skillstrainer bestätigt, indem er eine Einschätzung über verschiedene Voraussetzungen zur erfolgreichen Teilnahme am Skillstraining, wie z.B. Aufmerksamkeit, Zuverlässigkeit, Störanfälligkeit durch andere Patienten usw., abgibt. 
- Ausreichende Fähigkeit, erfolgreich an der Einzeltherapie teilzunehmen:

Diese Fähigkeit der Zusammenarbeit von Einzeltherapeut und Patient wird mit einem Videoband aus einem Einzelkontakt geprüft, in dem ein besonders schwieriges Thema besprochen wird (die schwierige Sitzung). Es wird dabei nicht verlangt, dass das besprochene Problem gelöst wird, sondern dass Patient und Einzeltherapeut dieses ohne dysfunktionales Vermeidungsverhalten wie dissoziieren, hinauslaufen, Streit anfangen oder Ähnliches besprechen können.

- In dem Gespräch mit dem Projektleiter soll die Verpflichtung zur Arbeit an den ACES-Zielen gestärkt werden und erste Vorstellungen zu angestrebten Arbeitstätigkeiten oder anderen Zielen können konkretisiert werden.

Wenn ein Patient seinen Antrag auf das zweite Behandlungsjahr erfolgreich gestellt hat, kann er sofort oder nach einer Pause mit der Teilnahme an den ACES-Angeboten beginnen. Wenn Teile des Antrags nicht erfolgreich durchlaufen wurden, besteht die Möglichkeit, diese so oft zu wiederholen, bis sie erfolgreich abgeschlossen wurden.

\section{Behandlungsangebote im ACES-Jahr}

Im ACES-Jahr werden Einzeltherapie, Skillsgruppe und die Möglichkeit zu Telefonanrufen in schwierigen Situationen in gleichem Umfang wie im ersten Behandlungsjahr angeboten. Die Möglichkeit zur Teilnahme an der Einzeltherapie ohne den Besuch des Skillstrainings ist in dem Konzept nicht vorgesehen. Der Fokus der Therapie im ACES-Jahr liegt neben der Aufrechterhaltung der im ersten Jahr erreichten Ziele in der Arbeit an 4 übergeordneten Zielbereichen:

- Den Anforderungen normativer, produktiver und belohnender Arbeit oder Ausbildung gerecht werden:

Unter «normativ» wird dabei verstanden, dass die Tätigkeit in einem Umfeld stattfindet, in dem sich alle so verhalten, als hätten sie keine psychischen Probleme. Belohnend sollte eine Tätigkeit durch das damit erzielte Einkommen sein oder durch die Aussicht auf einen Schul- oder Ausbildungsabschluss, der ein belohnendes Einkommen ermöglicht.

- Selbständigkeit und Autonomie in der Lebensführung: Eine selbständige Lebensführung unabhängig von Hilfen des Gesundheits- und Sozialsystems kann ein Gefühl von Freiheit und Unabhängigkeit bei vielen Entscheidungen und Entwicklungen begünstigen und damit das Selbstvertrauen und die Lebensqualität verbessern.

- Ein normatives soziales Netz unabhängig vom Gesundheitssystem:

Normative soziale Netze können in unterschiedlichen Bereichen wie Partnerschaften, Vereinszugehörigkeiten, Nachbarschaftskontakten, am Arbeitsplatz oder an anderen Orten aufgebaut werden. Die von den Patienten nach ihren eigenen Werten und Zielen gestalteten sozialen Beziehungen sollen tragfähig und außerhalb des Gesundheitssystems angesiedelt sein.

- Eigene Ziele des Patienten zur Erhöhung der Lebensqualität:

Persönliche Ziele zur Erhöhung der Lebensqualität können entsprechend der individuellen Werte der Patienten verfolgt werden.

Der therapeutische Fokus des zweiten Jahres verschiebt sich also in Richtung des Aufbaus zielorientierten und effektiven Handelns anstelle des Abbaus dysfunktionalen Handelns, was nicht nur eine stärkere Ressourcenorientierung in der Therapie beinhaltet, sondern auch zu einer nachhaltigen Verbesserung von Depressivität oder Identitätsstörungen beitragen kann.

In der ACES-Einzeltherapie werden grundsätzlich alle DBT-Strategien wie im ersten DBT-Jahr verwendet (dialektische Strategien, Commitmentstrategien, Validierungsstrategien, Verhaltensanalysen, Verhaltensbeobachtung mit Tagebuchkarten, usw.). Die Sitzung beginnt jedoch mit dem Überblick über die ACES-Tagebuchkarte, die sich in einer zusätzlich vorgesehenen Protokollierung der Schritte in Richtung der ACES-Ziele von der Tagebuchkarte aus dem ersten DBT-Jahr unterscheidet. Da in der Einzeltherapie wesentlich weniger Zeit für die Veränderung von therapiegefährdenden oder unkontrollierten problematischen Verhaltensweisen benötigt wird, liegt der Fokus der Therapie im zweiten Jahr vorwiegend auf der Verfolgung der ACES-Ziele oder in Hilfestellungen bei der Veränderung von ineffektivem Verhalten bei ihrer Verfolgung. Bei der Strukturierung von längerfristigen Zielen und den dafür notwendigen kurzfristigen Zwischenzielen und Aufgaben benötigen Patienten mit BPS Hilfe, da Hindernisse wie negative Grundannahmen über eigene Bewältigungskompetenzen oder Identitätsstörungen im Sinne einer ungenügenden Fähigkeit zur Selbsteinschätzung eigener Stärken, Schwächen oder Vorlieben das kontinuierliche Verfolgen der ACES-Ziele ohne Unterstützung verhindern können.

Beim Telefoncoaching im ACES-Jahr besteht für die Patienten die Möglichkeit, ihre Therapeuten in akuten Problemsituationen anzurufen und ein kurzes Coaching zu aktuellen Problemen bei der Verfolgung der ACES-Ziele zu erhalten.

Das ACES-Skillstraining ist in dem unpublizierten ACESManual von K.A. Comtois manualisiert beschrieben. Hier geht es nicht mehr um den Erwerb grundlegender Skills, sondern um 12 inhaltlich genau definierte und manualisierte Themenblöcke, die in einer Mischung aus Skillstraining und Psychoedukation vermittelt werden (Tab. 2). Zu Beginn jeder Skillsgruppe stellt jeder Patient anhand eines vorbereiteten Protokolls (das Check-in-Protokoll) in wenigen Minuten Fortschritte oder Hindernisse beim Verfolgen übergeordneter längerfristiger Ziele vor. Fortschritte werden in der Gruppe sozial verstärkt und Problemlösungen bei Misserfolgen werden in der Gruppe gefördert. Am Ende der «Check-in»- 
Runde setzt sich jeder Patient ein neues Wochenziel, um seinen längerfristigen Zielen oder persönlichen Projekten einen Schritt näher zu kommen. Im weiteren Verlauf des ACES-Skillstrainings werden dann die in der letzten Sitzung vereinbarten Hausaufgaben besprochen. Im Anschluss an die Pause werden in der zweiten Hälfte des Skillstrainings neue Inhalte besprochen.

Auch im zweiten Behandlungsjahr wird ein Kontingenzmanagement vereinbart:

- Kontingenzmanagement für versäumte Sitzungen:

Patienten, die 4 aufeinanderfolgende Sitzungen der Einzeltherapie oder 4 aufeinanderfolgende Sitzungen des Skillstrainings versäumen, werden von der Behandlung ausgeschlossen.

- Kontingenzmanagement für hochrangiges Stage-I-Verhalten: Patienten, die während des ACES-Jahres Rückfälle in schweres Stage-I-Verhalten erleben (z.B. suizidale Krisen, selbstverletzendes Verhalten, therapiezerstörendes Verhalten), erhalten eine zeitlich fest definierte und in der Regel mehrwöchige «Bewährungszeit», in der kein weiteres hochrangiges Stage-I-Verhalten auftreten darf. Andernfalls ist eine mehrwöchige Unterbrechung (Time-out) der Behandlung vorgesehen. Eine Rückkehr in die Therapie kann nach einem Time-out erfolgen, wenn mehrere Wochen hintereinander kein hochrangiges Stage-I-Verhalten mehr aufgetreten ist.

- Kontingenzmanagement für den Aufbau von Arbeitstätigkeit:

Sind folgende Bedingungen über einen Zeitraum von 4 aufeinanderfolgenden Wochen nicht erfüllt, ruht die Behandlung, bis die Bedingungen wieder für mindestens 1 Woche erfüllt sind:

Von Beginn des zweiten Behandlungsjahres an: $20 \mathrm{~h}$ geplanter Aktivitäten pro Woche und Vorbereitung von Arbeitstätigkeit oder Ausbildung.

Ab Monat 4: 20 h geplanter Aktivitäten, davon mindestens $10 \mathrm{~h}$ Arbeitstätigkeit oder Ausbildung.

$\mathrm{Ab}$ Monat 8: $20 \mathrm{~h}$ Arbeitstätigkeit oder Ausbildung nachgehen.

Im Kontingenzmanagement zählen alle Arbeitstätigkeiten oder schulischen oder beruflichen Ausbildungen, die regelmäßig, belohnend und sozialversichert sind und in einem normativen Umfeld stattfinden. Damit sind in erster Linie Tätigkeiten auf dem ersten Arbeitsmarkt gemeint oder solche Ausbildungsbemühungen, die auf den ersten Arbeitsmarkt führen. Klinische Erfahrungen zeigen, dass Borderline-Patienten sich aufgrund ihrer Identitätsstörungen und aufgrund geringen Selbsteffizienzerlebens stark an den Erwartungen ihrer soziokulturellen Umgebung orientieren. Aus diesem Grunde werden in dem Ansatz DBT-ACES keine Tätigkeiten gefördert, die innerhalb des Gesundheits- oder Sozialsystems vorwiegend im Patientenmilieu stattfinden. Aussichtsreicher erscheint es, dass die Teilnehmer des ACES-Jahres unter normativen Anforderungen Verhaltensweisen aufbauen und
Tab. 2. Ablauf des Curriculums im ACES-Skillstraining

\begin{aligned} & \hline Monat Behandeltes Modul \\ & \hline 1 Sich und andere verstärken \\ & 2 Achtsamkeit \\ & 3 Angstmanagement \\ & 4 Emotionsregulation \\ & 5 Dialektik \\ & 6 Stresstoleranz und radikale Akzeptanz \\ & 7 Zwischenmenschliche Fertigkeiten \\ & 8 Zeitmanagement \\ & 9 Depressionsmanagement \\ & 10 Perfektionismus \\ & 11 Nachfolgende Therapie \\ & 12 Ärgermanagement \\ & \hline\end{aligned}

verbessern, die auf dem ersten Arbeitsmarkt effektiv sind und auch langfristig eine belohnende und sozial abgesicherte Existenz begründen können.

\section{Effektivität von DBT-ACES}

Die Effektivität des ersten Jahres Standard-DBT wurde in mehreren kontrolliert randomisierten Studien unterschiedlicher Arbeitsgruppen belegt [Bohus et al., 2009]. Die psychopathologische Symptomatik wie suizidale und parasuizidale Handlungen, Selbstverletzungen, Drogeneinnahmen, Depressivität, Ängstlichkeit usw. konnte in allen Studien deutlich verbessert werden. Darüber hinaus zeigten Comtois et al. [2007], dass sich im ersten ambulanten Behandlungsjahr mit Standard-DBT nicht nur die stationären Behandlungszeiten schwer gestörter Borderline-Patienten um mehr als 2/3 reduzieren ließen, sondern sich auch die subjektive Lebensqualität der Betroffenen deutlich verbesserte. In einer gerade publizierten Prä-Post-Untersuchung [Comtois et al., 2010] zeigten Comtois und ihre Arbeitsgruppe, dass die Verbesserungen in der Psychopathologie und der subjektiven Lebensqualität im zweiten Behandlungsjahr mit DBT-ACES nicht nur aufrechterhalten werden konnten, sondern sogar noch gesteigert wurden. AuBerdem gelang es in dem ACES-Jahr, 80\% der teilnehmenden Patienten erfolgreich bei der Aufnahme einer belohnenden Tätigkeit oder schulischen Ausbildung mit mindestens $20 \mathrm{~h}$ Beschäftigungszeit pro Woche zu unterstützen. Von diesen $80 \%$ der Patienten, die am Ende der zweijährigen Behandlung einer mindestens halbtägigen Beschäftigung nachgingen, nahm etwa die Hälfte ihre Beschäftigung schon im ersten Behandlungsjahr auf. Das zeigt, dass die Behandlungsstufen nicht im Sinne zeitlich voneinander abgetrennter Behandlungsmodule missverstanden werden sollten, sondern als ineinanderfließende Schwerpunkte der Therapie. In der Katamnese ein Jahr nach dem Ende des ACES-Jahres blieben diese Behandlungserfolge stabil. Zu diesem Zeitpunkt nahmen nur noch 36\% der zuvor schwer erkrankten und häufig hospitalisierten Border- 
line-Patienten Leistungen des Gesundheitssystems in Anspruch, was sowohl aus gesundheitsökonomischer Sicht als auch im Hinblick auf die Ziele einer selbständigen Lebensführung mit der damit verbundenen Erhöhung der Lebensqualität ein äußerst ermutigendes Ergebnis ist.

\section{DBT-ACES im sozialpsychiatrischen Versorgungs- system in Deutschland}

DBT-ACES ist ein in den USA entwickelter Behandlungsansatz, dessen Einordnung in das deutsche sozialpsychiatrische Versorgungssystem Probleme aufwirft. Einerseits entspricht die verhaltenstherapeutische Behandlungsmethodik und die von Beginn an auf maximal 2 Jahre Behandlungszeit befristete vorübergehende Intervention einer Richtlinienpsychotherapie im Sinne einer verhaltenstherapeutischen Krankenbehandlung nach $\$ 27$ SGB-V. Andererseits werden insbesondere im zweiten Behandlungsjahr Behandlungsziele verfolgt, die im wesentlichen Partizipations- und Integrationsziele darstellen und in Deutschland im Sinne der Internationalen Klassifikation der Funktionsfähigkeit, Behinderung und Gesundheit (ICF) [WHO, 2001] Aufgaben der Grundversorgung sind. Linden [2008, 2009] weist darauf hin, dass ein Patient, der 6 Monate unter einer psychischen Erkrankung leidet oder zu leiden droht, nach dem SGB-IX als behindert oder von Behinderung bedroht gilt und sich daraus ein Anspruch auf Hilfen zur Partizipation ergibt. Hierfür ist im deutschen Versorgungssystem die kontinuierliche und zeitlich nicht befristete (sozial-) psychiatrische Versorgung vorgesehen, die dann durch unterschiedliche Integrationshilfen und Eingliederungsmaßnahmen ergänzt werden kann. Solche partizipationsorientierten Hilfen können z.B. stationär oder ambulant betreutes Wohnen, Maßnahmen zur Teilhabe am Arbeitsleben, medizinische oder berufliche Rehabilitationsmaßnahmen oder Maßnahmen der Familienhilfe beinhalten und werden von unterschiedlichen Kostenträgern wie z.B. der Krankenkasse, dem Sozialhilfeträger, dem Arbeitsamt oder der Rentenversicherung nach Prüfung entsprechender Anträge finanziert. Mehrheitlich beinhalten diese Hilfen heute allerdings keine störungsspezifische Verhaltenstherapie, in der Behandlungsstrategien sowohl für akute Symptomatik wie Suizidalität oder andere Stage-I-Probleme als auch für das Erreichen von Partizipations- und Integrationszielen angeboten werden. Daraus resultiert das Risiko eines Pendelns der Betroffenen zwischen vollstationärer psychiatrischer Akutbehandlung in Krisen und der Inanspruchnahme von partizipationsorientierten Hilfen, die dann nicht selten abgebrochen werden oder nicht mit ausreichender Kontinuität verfolgt werden können.

Eine Behandlung in dem Konzept DBT-ACES kann diese Problematik natürlich nicht bei allen Betroffenen nachhaltig lösen oder die Aufgaben der sozialpsychiatrischen Grundversorgung in Deutschland bei den oft jahre- oder jahrzehntlangen Krankheitsverläufen von Patienten mit BPS übernehmen.
Für Betroffene, die den Versuch wagen möchten, Hilfen des Versorgungssystems abzubauen und eine belohnende Tätigkeit, eine normative soziale Integration und die Unabhängigkeit vom Versorgungssystem zu erreichen, bietet DBT-ACES allerdings sehr spezifische Unterstützungsmöglichkeiten an. Die Einzelpsychotherapie kann in diesem Konzept in Deutschland nur als zeitlich befristete «Add-on-Behandlung» $\mathrm{zu}$ den Behandlungsmöglichkeiten der Grundversorgung konzipiert werden und entspricht einer Richtlinienpsychotherapie. Die Psychotherapierichtlinien sprechen nicht gegen eine solche Einordnung, da nach $\$ 22$ Absatz 5 auch die Partizipationsziele in einer Richtlinienpsychotherapie berücksichtigt werden dürfen (oder vielleicht sogar müssen), wenn «Verhaltensweisen, die als psychosoziale Störungen in Erscheinung treten, ... Ausdruck einer psychischen Erkrankung sind» [Gemeinsamer Bundesausschuss, 2009]. Die pharmakologische Therapie der Patienten, das Skillstraining sowie die sozialarbeiterische Unterstützung kann im deutschen Versorgungssystem von der ambulanten psychiatrischen Behandlung bzw. durch die Angebote sozialpädagogischer Integrationshilfen realisiert werden. Obwohl diese multimodale und multidisziplinäre Zusammenarbeit in den wöchentlichen Teamsitzungen in der ambulanten Behandlung aus praktischen und institutionellen Gründen schwer zu organisieren ist, ist sie unverzichtbarer Bestandteil der DBT. Die Verbesserung der Abstimmung und des Wissens über Behandlungsschritte der anderen Berufsgruppen im Bezug auf die Behandlung eines einzelnen Patienten ist dabei nicht der einzige Vorteil. Betroffenen kann die Fortführung der sozialpädagogischen und psychiatrischen Behandlungen auch nach einem Ausscheiden aus der Behandlung mit DBT-ACES im Sinne der sozialpsychiatrischen Grundversorgung kontinuierlich gewährleistet werden, insbesondere wenn die anspruchsvollen Ziele des zweiten DBT-ACES-Jahres nicht (oder nicht innerhalb der konzeptionell vorgesehenen Zeiträume) erreicht werden.

\section{Aktuelle Aktivitäten und Ausblick}

Derzeit evaluiert die Arbeitsgruppe um Comtois in Seattle DBT-ACES in einer methodisch anspruchsvollen kontrolliert randomisierten Studie. In Deutschland wird die Therapie seit 2010 in der LWL-Klinik Lengerich ambulant mit einem Jahr Standard-DBT und der Möglichkeit zu einem zweiten Jahr DBT-ACES angeboten und in einer Prä-Post-Anwendungbeobachtung evaluiert. Erste Erfahrungen aus der Therapie, in der derzeit 20 Patienten mit ambulanter Standard-DBT im ersten Behandlungsjahr und DBT-ACES im zweiten Behandlungsjahr versorgt werden, sind dabei äußerst ermutigend.

\section{Disclosure Statement}

Es bestehen keine Interessenkonflikte. 


\section{Literatur}

Barnicot K, Katsakou C, Marougka S, Priebe S: Treat-

ment completion in psyochtherapy for borderline personality disorder - a systematic review and metaanalysis. Acta Psychiatr Scand 2011;123:327-338.

Bohus M, Buchheim P, Doering S, Herpertz SC, Kapfhammer HP, Linden M, Müller-Isberner R, Renneberg B, Saß H, Schmitz B, Schweiger U, Resch F, Tress W: S2 Praxisleitlinien in Psychiatrie und Psychotherapie. Band 1 Behandlungsleitlinie Persönlichkeitsstörungen. Darmstadt, Steinkopff, 2009.

Bohus M, Wolf M: Interaktives Fertigkeitentraining für Borderline-Störungen. Stuttgart, Schattauer, 2009.

Coid J, Yang M, Tyrer P, Roberts A, Ullrich S: Pre-

valence and correlates of personality disorder in Great Britain. Br J Psychiatry 2006;188:423-431.

Comtois KA, Elwood LM, Holdcraft LC, Smith WR,

Simpson TL: Effectiveness of dialectical behavioral therapy in a community mental health center. Cogn Behav Pract 2007;14:406-414.

Comtois KA, Kerbrat AH, Atkins DC, Harned MS

Elwood L: Recovery from disability for individuals with borderline personality disorder: a feasibility trial of DBT-ACES. Psychiatr Serv 2010;61:11061111.

Gemeinsamer Bundesausschuss: Richtlinie des Gemeinsamen Bundesausschusses über die Durchführung der Psychotherapie (Psychotherapie-Richtlinie) in der Fassung vom 19.02.2009. Bundesanzeiger 2009;58:1399.

Jerschke S, Meixner K, Richter H, Bohus M: Zur

Behandlungsgeschichte und Versorgungssituation von Patientinnen mit Bordeline Persönlichkeitsstörungen in der Bundesrepublik Deutschland. Fortschr Neurol Psychiatr 1998;66:545-552.
Lenzenweger MF, Lane MC, Loranger AW, Kessler RC: DSM-IV personality disorders in the National Comorbidity Survey Replication. Biol Psychiatry 2007;62:553-564.

Linden M: Was ist Psychotherapie gemäß SGB IX? Verhaltenstherapie 2008;18:144-145.

Linden M: Rehabilitationspsychotherapie. Definition, Aufgaben uund Organisationsformen nach ICF und SGB IX. Prax Klin Verhaltensmed Rehabil 2009;84: 137-142.

Linehan MM: Cognitive-Behavioral Treatment of Borderline Personality Disorder. New York, Guilford press, 1993a.

Linehan MM: Skills Training Manual for Treating Borderline Personality Disorder. New York, Guilford press, $1993 \mathrm{~b}$.

Paris J, Zweig-Frank H: A 27-year follow-up of pa-

tients with borderline personality disorder. Compr Psychiatry 2001;42:482-487.

Soeteman DI, Hakkaart-van Roijen L, Verheul R,

Busschbach JV: The economic burden of personality disorders in mental health care. J Clin Psychiatry 2008;69:259-265.

Steil R, Dyer A, Priebe K, Kleindienst N, Bohus M: Dialectical behavior therapy for posttraumatic stress disorder related to childhood sexual abuse: a pilot study of a intensive residential treatment program. J Traumatic Stress;24:102-106.

Trull TJ, Jahng S, Tomko RL, Wood PK, Sher KJ: Re-

vised NESARC personality disorder diagnoses: gender, prevalence, and comorbidity with substance dependence disorders. J Pers Disord 2010;24:412426
Van Asselt A, Dirksen C, Arntz A, Severens J: The cost of borderline personality disorder: societal cost of illness in BPD patients. Europ Psychiatry 2007;6: 354-361.

Winograd G, Cohen P, Chen H: Adolescent border-

line symptoms in the community: prognosis for functioning over 20 years. J Child Psychol Psychiatry 2008;49:933-941.

World Health Organization: International Classification of Functioning, Disability and Health (ICF) Genf, WHO, 2001

Zanarini MC, Frankenburg FR, Hennen J, Reich DB,

Silk KR: The McLean Study of Adult Development (MSAD): overview and implications of the first six years of prospective follow-up. J Pers Disord 2005; 19:505-523.

Zanarini MC, Frankenburg FR, Reich DB, Silk KR,

Hudson JI, McSweeney LB: The subsyndromal phenomenology of borderline personality disorder: a 10-year follow-up study. Am J Psychiatry 2007; 164:929-935.

Zanarini MC, Frankenburg FR, Bradford Reich D,

Fitzmaurice G: Time to attainment of recovery from borderline personality disorder and stability of recovery: a 10-year prospective follow-up study. Am J Psychiatry 2010a;167:663-667.

Zanarini MC, Frankenburg FR, Bradford Reich D,

Fitzmaurice G: The 10-year course of psychosocial functioning among patients with borderline personality disorder and axis II comparison subjects. Acta Psychiatr Scand 2010b;122:103-109. 